\title{
Glaucoma Detection in Retinal Images using Automatic Thresholding and Marker - Controlled Watershed Transformation
}

\author{
Manikandan T, Satheesh Kumar S, Joshua Kumaresan S, Shobana Priya M S, Priyanka R
}

\begin{abstract}
The eye is an organ in human, responsible for the vision. However, it gets affected by the diseases. Glaucoma is a one such eye disease. It develops in the eye due to the increase in intra-ocular pressure. If glaucoma is not treated in its initial stage, leads for permanent vision loss. This work is aimed to develop a computer aided diagnosis system for glaucoma detection in fundus retinal images. In this paper, we presented a method to automatically outline the optic disc in a retinal image by automatic thresholding technique. The optic cup is segmented based on marker-controlled automatic watershed transformation. The optic cup to disc ratio $(O C D R)$ is calculated, to show the presence of glaucoma. The proposed work is assessed with 15 normal retinal images and 15 retinal images with glaucoma, retrospectively collected from the Annai Eye Clinic, Chennai. To validate the system performance, obtained results were compared with the ophthalmologist results (taken as the gold standard). The experimental results show that, the proposed work is potential for the glaucoma detection.
\end{abstract}

Keywords: Fundus retinal image, Optic cup, Optic disc, Thresholding and Watershed transformation.

\section{INTRODUCTION}

Gla laucoma is an eye disease caused by increase in intra-ocular pressure [1]. Increase in intra-ocular pressure in the eye is due to the increase in volume of aqueous humor fluid secretion. The increased fluid to be drained out through the drainage canals of the eye. Glaucoma is developed when path for the excess fluid is blocked. Due to this, the aqueous humor fluid cannot drain away normally and the pressure in the eye increases gradually. The optic nerve in the eye is damaged [2]. This causes enlargement of optic cup, which in turn leads for vision loss, if not treated. The changes in optic cup is a vital sign for the glaucoma detection in clinical therapy. The computer aided diagnosis systems (CAD) are developed to detect the abnormalities in medical images [3, 4]. Hence, the design of CAD systems are necessary to identify the changes in the structure of optic cup, which may help ophthalmologist to diagnose the presence of glaucoma.

Revised Manuscript Received on November 12, 2019

* Correspondence Author

Manikandan T, Professor, Department of ECE, Rajalakshmi Engineering College, Chennai, India,Email: manikandan.t@rajalakshmi.edu.in

Satheesh Kumar S, Assistant Professor,Department of ECE, Sri Krishna College of Engineering and Technology, Coimbatore, India, Email: ssatheeshkumarpsg@gmail.com

Joshua Kumaresan S, Associate Professor,Department of ECE, R.M.K Engineering College, Chennai, India, Email: skn.ece@rmkec.ac.in

Shobana Priya M S, Assistant Professor, Department of ECE, Saveetha School of Engineering, Chennai, India, Email:shobanapriyams.sse@saveetha.com

Priyanka R, Assistant Professor, Department of ECE, Saveetha School of Engineering, Chennai, India, Email:priyankar.sse@ saveetha.com
Many studies have been conducted for the glaucoma detection from retinal fundus images. The glaucoma detection system reported by $\mathrm{Man} \mathrm{Hu}$ et al for the optic cup segmentation utilized both color difference information and vessel bends information to find the boundary of the optic cup [5]. Their experiment analysis described that the optic cup boundaries were more consistent than the existing methods. Anum Abdul Salam et al demonstrated a novel algorithm for the glaucoma detection based on a fusion of cup to disc ratio and hybrid textural and intensity features [6]. Their study concluded that, the fusion of CDR with hybrid features improved the sensitivity, specificity and accuracy of the computer aided diagnosis system. Fauzia Khan et al described an automatic system for the glaucoma detection by extracting two features from the retinal fundus images [7]. They extracted CDR and neuroretinal rim ratio and nasal quadrants for the classification of retinal images under normal and glaucoma condition. Anindita Septiarini et al developed an automated glaucoma detection system using the statistical features [8]. The features are extracted from optic nerve head in the fundus retinal images. The features are extracted by feature extraction method and feature selection by correlation feature selection method. The k-nearest neighbor algorithm is applied on the selected features to detect the glaucoma from the retinal images.

This paper is focuses on the optic disc and optic cup segmentation in fundus retinal images. The optic disc is segmented by adaptive thresholding technique and optic cup is segmented by marker-controlled watershed transformation technique. Finally the OCDR is calculated to show the presence of glaucoma in the fundus retinal images

\section{PROPOSED METHOD}

The proposed method uses two stages for the detection of glaucoma in the fundus retinal images. In the first stage, the optic disc is segmented by automatic thresholding technique. In the second stage, the optic cup is segmented by marker-controlled watershed transformation approach. After the segmentations of optic disc and optic cup, the OCDR is computed to detect the presence of glaucoma

\section{A. Optic Disc Segmentation by Thresholding}

Segmentation is a technique, which partition the given image into its constituent regions [9]. After the Region of Interest (ROI) is isolated, the segmentation will be stopped. Here, the ROI is optic disc in the retinal images. The optic disc is segmented by automatic thresholding technique, which is as follows: 
- Find the histogram of the retinal image.

- Select the threshold value automatically from the histogram in order to segment the optic disc.

The optic disc region inthe retinal image has similar gray level values. The optic disc is segmented by selecting proper gray-level value (threshold). Here threshold is computed from image histogram automatically to segment the optic disc from the retinal images. The figure 1 gives the image histogram (distribution of gray level values) of the fundus retinal image. From figure 1, it is clear that, the gray level values for background, optic disc and optic cup will fall in three dominant regions. Thus, the optic disc is segmented by automatic thresholding technique

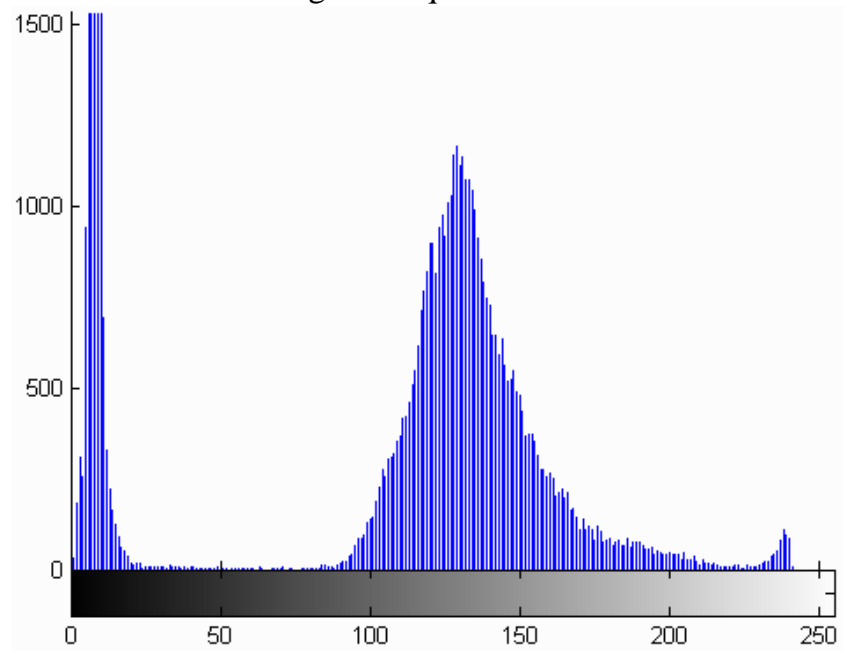

Fig. 1 Histogram of Retinal Image

\section{B. Segmentation of Optic Cup by Marker - Controlled Watershed transformation}

The glaucoma detection is carried out by analysing the optic cup. The optic cup will be enlarged under glaucoma condition. If severe the glaucoma, severe will be enlargement of optic cup and vice-versa. Hence, it is necessary to segment the optic cup after the segmentation of optic disc. The optic cup size is varies from patient to patient depending on the progression of glaucoma; its diameter lies between 30 and 50 pixels in 256X256 size images.

\section{The Optic cup localization}

The fundus retinal image is first filtered and watershed transformation is applied to locate the optic cup boundaries. The filtering operation on the retinal image is performed by $3 \times 3$ mean filter. The optic cup represents a bright area, and as blood vessels emerge dark in gray level in retinal images. This variation is first eliminated by closing morphological operation before applying the watershed segmentation.

In green channel, the optic cup appears as the brightest part and its contours looks most continuous and most contrasted against background. Hence, green channel output is further analysed for optic cup localization. To locate the optic cup, find out the maximum intensity in the fundus retinal image. This works well for the retinal images with normal images (no exudates and drusen). The optic cup localization is done by a shade correction operator to eliminate slow background variations, which reduces the contrast of exudates and drusen. The locus of the optic disc, $\mathrm{c}(\mathrm{x}, \mathrm{y})$ is approximated by calculating the maximum of the shade corrected image.

\section{The marker-Controlled Watershed Transformation}

The fundus retinal image contains many objects of different sizes that are touching each other. The watershed segmentation is used to subdivide the touching objects. As the eye image consists of various touching objects such as optic cup, optic disc, vessels it watershed segmentation is the right candidate for this segmentation.

The morphological closing operator and opening operators are applied on the input image before watershed segmentation, to eliminate large gray level variations and large peaks. This results, modification in the shape of papillary region. Therefore, morphological reconstruction is performed by dilation $[10,11]$. The internal and external markers are imposed before performing the watershed segmentation. The locus of optic cup is selected as internal marker and the external marker is square containing the optic cup region [12].

The watershed transformation returns non-negative values for the pixels in the watershed region. The pixel values are labelled as zero, which do not fall in the watershed region. Thus, the gray scale of the pixels are modified by watershed function. The marked region will have regional minima and other pixels are pushed up to eliminate other regional minima. As a result, the optic cup is segmented by marker-controlled watershed transformation.

\section{CALCULATION OF OPTIC CUP-TO-DISC RATIO}

The OCDR is calculated by counting total number of pixels in the segmented optic cup and disc regions. The OCDR is obtained by dividing the number of pixels in the optic cup and number of pixels in the optic disc. The calculated OCDR is 0.3 then the retinal image is said to be normal eye images. If OCDR exceeds more than 0.3 , the retinal image said to be under glaucoma condition. The severity of the progression of glaucoma is found on the OCDR value. If higher the OCDR value, the progression of the glaucoma is severe and vice-versa.

\section{RESULTS AND DISCUSSION}

The proposed approach was analysed with fundus retinal images retrospectively collected from Annai Eye Clinic, Chennai. The collected database had 15 normal retinal images and 15 fundus retinal images with glaucoma condition. For all the 30 images, first, optic disc was segmented. Second, the optic cup was segmented.

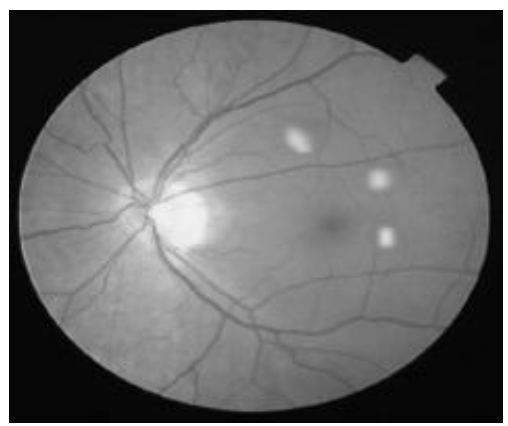

Fig. 3 Input image 
Then, the optic cup-disc ratio (OCDR) is computed to find the presence of glaucoma. The optic cup-disc ratio is calculated to be greater than 0.3 then glaucoma will be detected from the input fundus image. Otherwise, the input fundus image is found to be normal.

The Fig 3 shows the input fundus image. The segmented optic disc is shown in Fig 4. Fig 5 shows the segmented optic cup and Fig 6 shows the calculation of optic cup-disc ratio. The presence of glaucoma is found based on OCDR. For the given input image (Fig3) optic cup-disc ratio was found to be 0.6912 . Hence the glaucoma is present.

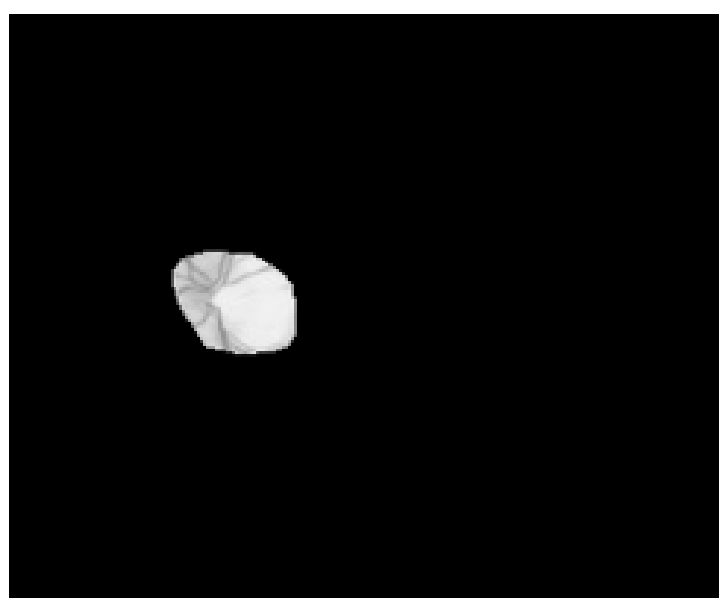

Fig. 4 Segmented Optic Disc

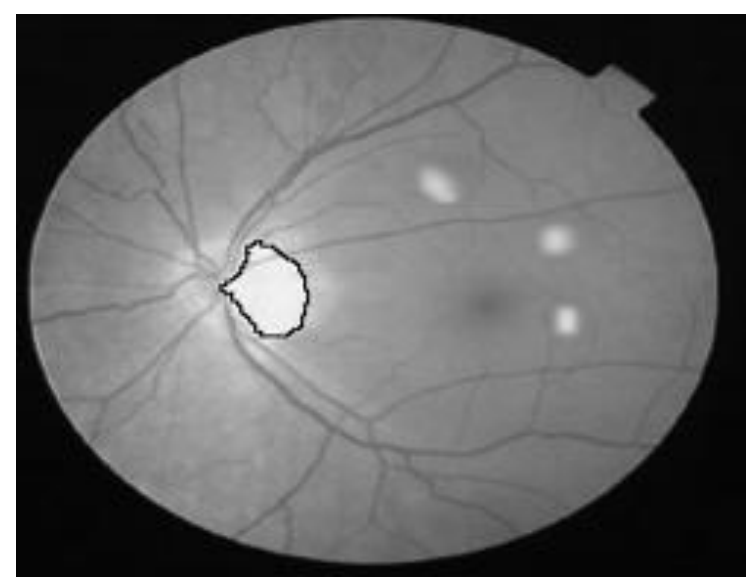

Fig. 5 Segmented optic cup

\begin{tabular}{|c|c|}
\hline A. NATLB & 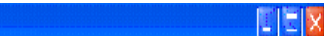 \\
\hline \multicolumn{2}{|l|}{ 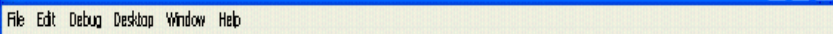 } \\
\hline 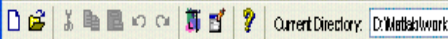 & *... 目 \\
\hline \multicolumn{2}{|l|}{ 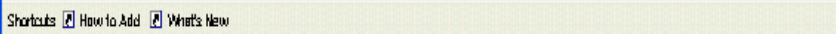 } \\
\hline \multicolumn{2}{|l|}{ Nomber of pixels present in disc area $=1490$} \\
\hline \multicolumn{2}{|l|}{ number of pixels present in cup area $=1030$} \\
\hline Cup to d1se rat10 $=0.6912$ & \\
\hline$\gg$ & \\
\hline
\end{tabular}

Fig. 6 Calculation of optic cup-disc ratio

Tables 1 and Table 2 shows that, the calculated optic cup-disc ratio for the fundus retinal images under normal and glaucoma conditions, respectively. To validate the obtained results, the optic cup-disc ratio calculated by the ophthalmologist was taken as the gold standard.

Table I Optic cup-disc ratio for normal fundus retinal images

\begin{tabular}{|c|c|c|}
\hline $\begin{array}{c}\text { Patient } \\
\text { id\# }\end{array}$ & $\begin{array}{c}\text { Optic-cup ratio } \\
\text { calculated by } \\
\text { ophthalmologist }\end{array}$ & $\begin{array}{c}\text { Optic-cup ratio calculated } \\
\text { by proposed approach }\end{array}$ \\
\hline$\# 1$ & 0.20 & 0.22 \\
\hline$\# 2$ & 0.28 & 0.28 \\
\hline$\# 3$ & 0.22 & 0.21 \\
\hline$\# 4$ & 0.18 & 0.19 \\
\hline$\# 5$ & 0.19 & 0.20 \\
\hline$\# 6$ & 0.25 & 0.23 \\
\hline$\# 7$ & 0.27 & 0.28 \\
\hline$\# 8$ & 0.21 & 0.21 \\
\hline$\# 9$ & 0.22 & 0.20 \\
\hline$\# 10$ & 0.16 & 0.18 \\
\hline$\# 11$ & 0.15 & 0.15 \\
\hline$\# 12$ & 0.17 & 0.20 \\
\hline$\# 13$ & 0.26 & 0.28 \\
\hline$\# 14$ & 0.28 & 0.27 \\
\hline$\# 15$ & 0.23 & 0.22 \\
\hline
\end{tabular}

Table II Optic cup-disc ratio for normal fundus retinal images

\begin{tabular}{|c|c|c|}
\hline $\begin{array}{c}\text { Patient } \\
\text { id\# }\end{array}$ & $\begin{array}{c}\text { Optic-cup ratio } \\
\text { calculated by } \\
\text { ophthalmologist }\end{array}$ & $\begin{array}{c}\text { Optic-cup ratio calculated } \\
\text { by proposed approach }\end{array}$ \\
\hline$\# 1$ & 0.35 & 0.36 \\
\hline$\# 2$ & 0.32 & 0.34 \\
\hline$\# 3$ & 0.68 & 0.68 \\
\hline$\# 4$ & 0.72 & 0.70 \\
\hline$\# 5$ & 0.58 & 0.56 \\
\hline$\# 6$ & 0.45 & 0.46 \\
\hline$\# 7$ & 0.52 & 0.51 \\
\hline$\# 8$ & 0.74 & 0.76 \\
\hline$\# 9$ & 0.67 & 0.64 \\
\hline$\# 10$ & 0.33 & 0.36 \\
\hline$\# 11$ & 0.36 & 0.35 \\
\hline$\# 12$ & 0.40 & 0.39 \\
\hline$\# 13$ & 0.64 & 0.64 \\
\hline$\# 14$ & 0.35 & 0.34 \\
\hline$\# 15$ & 0.52 & 0.50 \\
\hline
\end{tabular}

From the above tables it is evident that, the calculated OCDR by the proposed method is very closure to the OCDR calculated by the ophthalmologist 


\section{CONCLUSION}

Optic cup localization and segmentation is necessary for the glaucoma detection. The progression of optic cup in the eye takes place under glaucoma condition. The optic cup-disc ratio measurement is very important to identify the presence and severity of the glaucoma. In the proposed work, the optic disc and optic cup were segmented by thresholding technique and watershed transformation, respectively. Then, the OCDR was calculated to find the presence of the glaucoma. Glaucoma is detected from the fundus retinal image, if the OCDR is greater than 0.3 .

This work demonstrated an automatic detection of glaucoma based on only the optic cup-disc ratio measurement. In future, the other parameters such as neuroretinal rim width, neuroretinal area, and vertical cup-disc ratio may also be calculated to indicate the progressive enlargement of the optic cup. The progression of glaucoma may be calculated by 3-D reconstruction.

\section{REFERENCES}

1. M. D. Abramoff, W. L. M. Alward, E. C. Greenlee, L. Shuba, C. Y.Kim, J. H. Fingert, and Y. H. Kwon, "Automated segmentation of the optic disc from stereo color photographs using physiologically plausible features," Investigative Ophthalmology \& Visual Science, vol. 48, pp. 1665-1673, 2007.

2. S. Bibin and T. Manikandan, 'Blood vessel detection from fundus image for diabetic retinopathy patients using SVM,'in International Journal of Engineering and Technology, E-ISSN: 2278-018, vol. 3, no. 5, pp. $1248-1253,2014$

3. T. Manikandan and N. Bharathi, 'Lung cancer detection using fuzzy auto-seed cluster means morphological segmentation and SVM classifier', Journal of medical Systems (A Springer journal), E-ISSN: 1573-689X, P-ISSN: 0148-5598, vol. 40, no. 7, pp. 1-9, 2016.

4. T. Manikandan and N. Bharathi, 'A survey on computer-aided diagnosis systems for lung cancer detection', International Research Journal of Engineering and Technology, E-ISSN: 2395-0056, P-ISSN: 2395-0072, vol. 3, no. 5, pp. 1562-1570, 2016.

5. Man $\mathrm{Hu}$, Chenghao Zhu, Xiaoxing $\mathrm{Li}$ and Yongli $\mathrm{Xu}$, "Optic cup segmentation from fundus images for glaucoma diagnosis', Bioengineered, vol. 8(1), pp. 21-28, 2017.

6. Anum Abdul Salam, M. Usman Akram, Kumaran Wazir, Syed Muhammad Anwar and Muhammad Majid, "Autonomous Glaucoma detection from fundus image using cup to disc ratio and hybrid features', proceedings of 2015 IEEE International Symposium on Signal Processing and Information Technology (ISSPIT), 2015 DOI: 10.1109/ISSPIT.2015.7394362.

7. Fauzia Khan, Shoaib A. Khan, Ubaid Ullah Yasin, Ihtisham ul Haq, Usman Qamar, "Detection of Glaucoma Using Retinal Fundus Images", The 2013 Biomedical Engineering International Conference (BMEiCON-2013), DOI: 10.1109/BMEiCon.2013.6687674.

8. Anindita Septiarini, Dyna M. Khairina, Awang H. Kridalaksana, "Automatic Glaucoma Detection Method Applying a Statistical Approach to Fundus Images', Healthcare informatics research, vol. 24(4): pp. 335-345, 2018. DOI: 10.4258/ hir.2018.24.1.53.

9. K. Iyshwerya, B. Janani, S. Krithika and T. Manikandan, 'Defect detection algorithm for high speed inspection in machine vision,' in International Conference on Smart Structures \& Systems at Saveetha Engineering College, March 28-29, 2013, DOI: 10.1109/ ICSSS.2013.6623010.

10.K. Akita, H. Kuga. A computer method of understanding ocular fundus images. Pattern Recognition, 15(6):431-443, 2012.

11.Katarzyna stpor, Leslaw Pawlaczyk, Radim Chrastek, Automatic segmentation and classification, IEEE Conference on Computer Vision and Pattern Recognition, pp. 800-815, 2002.

12.Rafel C. Gonzalez, Richard E. Woods and Steven L. Eddins. Digital Image Processing Using Matlab. Pearson Education (Singapore) Pte. Ltd., 2005

\section{AUTHORS PROFILE}

Dr. T. Manikandan is a Professor working at Rajalakshmi Engineering

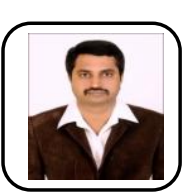
College, Thandalam, Chennai. He has completed his Ph.D titled "A study on computer-aided diagnosis systems for lung cancer detection and its three dimensional visualization using machine learning techniques" at Anna University, Chennai during January, 2017. He did his bachelor's degree in Engineering from Vellore Engineering College, Chennai under Madras University \& master's degree in Engineering from College of Engineering, Anna University, Guindy, Chennai during 1998 \& 2007, respectively. He is having 21 years of teaching experience which includes both UG and PG. He also guided many projects at UG \& PG levels. He has 50 papers in refereed international journals and conferences in his credit. He is serving as editorial board member for various international journals such as International journal of Biomedical and Healthcare Sciences, Archives of General Internal Medicine, Journal of Cancer Diagnosis, Journal of Medical and Clinical Oncology, Journal of Clinical Epigenetics and Integrative Cancer Biology and Research.

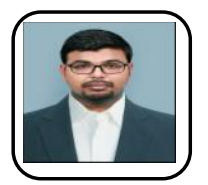

Satheesh Kumar S currently working as Assistant Professor at Sri Krishna College of Engineering and Technology, Coimbatore. He accomplished his Master of Engineering in the field of Communication Systems from PSG College of Technology, Coimbatore and B.E. from Anna University, Chennai. He is also a Life time member of IETE and ISRD. Having more than 4 years of teaching experience, he is acting as a board member for some Private Engineering Institutions in Coimbatore. He is also a recipient of various honors and faculty awards in his teaching fraternity. He also published over 20 technical articles in various International and national journals and filed a patents in his research field.His research focus include Electromagnetics, Microwave Engineering, Speech Processing and Block Chain technology.

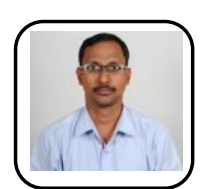

Dr.S.Joshua Kumaresan is working as Associate professor in the department of ECE of R.M.K Engineering college. He completed his $\mathrm{Ph} . \mathrm{D}$ in the area of image processing in 2018, M.E (VLSI Design) in 2007 both from Anna university Chennai. He also completed M.S (Electronics and Control) from BITS, Pilani in 1999 and B.E (ECE) in 1994 from Bharathidasan University. He has 23 years of teaching experience which includes both UG and PG. and 1 year he worked in an industry as R\& D Engineer. He published more than 20 papers in international journal and conferences.He published books on Electromagnetic Fields, Digital Electronics, Digital Principles system design and Digital logic circuits. . He guided many projects at UG \& PG levels. He also mentored and guided many students for various competitions like Smart India Hackathon, Arm design contest, India Innovation challenge design contest 2018 etc. He organized many college level National conferences and symposiums. He is a member of professional societies IEI, ISTE, ISRO-ISSE, and IACSIT.

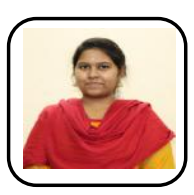

Mrs. M S. Shobana Priya is a Assistant Professor working at Saveetha School of Engineering, Thandalam, Chennai. She is currently pursuing her Ph.D in Machine learning at Saveetha School of Engineering, SIMATS, Thandalam since June, 2018. She did her bachelor's degree in Engineering from Jaya Engineering College, Thiruniravur under Anna University \& master's degree in Engineering from College of Engineering, Anna University, Guindy during 2011 \& 2015, respectively. She is having 1.5 years of teaching experience in UG. She also guided many projects at UG levels.

R.Priyanka pursued her B.E Degree in Electronics and Communication Engineering in 2015 and M.E degree in Applied Electronics from R.M.K Engineering College in 2017.She is pursuing her Ph.D degree and working as an Assistant Professor in ECE Department at Saveetha school of Engineering. She has a teaching experience of 2 years. Her area of research includes Machine learning and Artificial Intelligence. 